\title{
Burn-in for a time-transformed exponential model
}

\author{
Andrzej Giniewicz • Alicja Jokiel-Rokita
}

Received: 13 December 2010 / Published online: 19 February 2012

(C) The Author(s) 2012. This article is published with open access at Springerlink.com

\begin{abstract}
The problem of the optimal duration of a burn-in experiment is considered in the case of simultaneous testing $n$ components with the conditionally independent time-transformed exponential life-times, given an unknown parameter. The explicit solution is derived by reformulation of the problem considered to an optimal stopping problem for a suitable defined three-dimensional Markov process and reduction to a free-boundary problem.
\end{abstract}

Keywords Burn-in · Free-boundary method - Optimal stopping · Time-transformed exponential distribution

\section{Introduction}

Burn-in is widely used in engineering reliability, statistical simulations, and medical sensitivity testing. Traditionally, it is used to consume a product's life until it reaches the first change-point where the failure rate of the product is reduced to a low level. Various criteria of optimality are used in practice. Cost minimization is mostly considered, especially in the case of decreasing failure rate of the life-times. Reward maximization is considered by Aven and Jensen (1999), where a semimartingale approach for solving a burn-in problem is applied. Mi (1995) has shown that if the component has a bathtub failure rate function, then the mean residual life (MRL) of the component has an upside-down bathtub shape. Therefore, another approach for an optimal burn-in policy is to maximize the MRL for the bathtub-shaped failure rate function. The case when lifetime distributions have a unimodal failure rate function (where the failure

\footnotetext{
A. Giniewicz · A. Jokiel-Rokita $(\varangle)$

Institute of Mathematics and Computer Science, Wrocław University of Technology,

Wybrzeże Wyspiańskiego 27, 50-370 Wrocław, Poland

e-mail: alicja.jokiel-rokita@pwr.wroc.pl
} 
rate function increases to attain its maximum at a critical time, and then decreases) was considered by Chang (2000). For detailed reviews of burn-in models and methods we refer, for example, to Leemis and Beneke (1990), Kececioglu and Sun (1997), Block and Savits (1997) and Lai and Xie (2006).

In this paper the optimal burn-in time is derived under the criterion of cost minimization in testing $n$ items $I_{1}, \ldots, I_{n}$. To save time, all items are tested simultaneously and the experiment can be stopped at any time. Let $T_{1}, \ldots, T_{n}$ denote the lifetimes of the items $I_{1}, \ldots, I_{n}$, respectively. If the observation process is stopped at time $t$, the values of $T_{1}, \ldots, T_{n}$ not exceeding $t$ are exactly known, whereas the other $T_{i}$ are only known to be larger than $t$. The observations available in this way are sometimes called in literature as longitudinal observational data (see Arjas 1989). If the component fails during the test it is discarded, otherwise-it is delivered to operations. We fix a cost $c>0$ to be paid for each component that fails during the test, and a cost $C(r), r \in[0, \infty)$, for each component that is put into operation and has an operative lifetime $r$. We assume that the function $C(r)$ is a right continuous, non-increasing and bounded function such that

$$
\lim _{r \rightarrow \infty} C(r)<c
$$

and

$$
\lim _{r \rightarrow 0^{+}} C(r)>c
$$

Let $T_{(1)}, \ldots, T_{(n)}$ be the order statistics of $T_{1}, \ldots, T_{n}$, and, for each $t \geq 0$, let

$$
H_{t}:=\sum_{i=1}^{n} \mathbf{1}_{[0, t]}\left(T_{i}\right)
$$

be the number of components that have failed up to time $t$. Then, if the process is stopped at time $\sigma$ (possibly depending on the outcome of the experiment), one expects to pay a cost

$$
J(\sigma):=\mathrm{E}\left[c H_{\sigma}+\mathbf{1}_{\left\{H_{\sigma} \leq n-1\right\}} \sum_{j=H_{\sigma}+1}^{n} C\left(T_{(j)}-\sigma\right)\right] .
$$

We will denote by

$$
\mathscr{F}_{t}:=\sigma\left(T_{1} \wedge t, \ldots, T_{n} \wedge t\right)
$$

and by $\mathscr{T}$ the set of all stopping times relative to $\left(\mathscr{F}_{t}\right)_{t \geq 0}$. By the burn-in problem we mean the problem of choosing a stopping time $\sigma^{*} \in \mathscr{T}$ such that

$$
J\left(\sigma^{*}\right)=\inf _{\sigma \in \mathscr{T}} J(\sigma) .
$$


The burn-in problem is often considered under the assumption that the lifetimes are independent, identically distributed (i.i.d.) random variables, what implies that they are exchangeable, infinitely extendible. In this paper we solve the burn-in problem under the assumption that the random variables $T_{1}, \ldots, T_{n}$ are conditionally independent time-transformed exponential distributed, given an unknown parameter $\lambda$, i.e. the conditional distribution of the lifetimes is given by the density

$$
f(t \mid \lambda):=\lambda s^{\prime}(t) \exp \{-\lambda s(t)\} \mathbf{1}_{(0, \infty)}(t)
$$

where $s(t)$ is a known, strictly increasing and differentiable function with $s(0)=0$ and $\lim _{t \rightarrow \infty} s(t)=\infty$. It is assumed that the parameter $\lambda$ is a realization of a random variable from a distribution $\pi_{0}$ on $[0, \infty)$ which, taking a Bayesian viewpoint, can be seen as a prior knowledge about the unknown parameter $\lambda$. By de Finetti's theorem (de Finetti 1937) conditionally i.i.d. random variables given some parameter are also exchangeable, infinitely extendible. The assumption of exchangeability for the lifetimes of the units in a production line adequately expresses the idea that the production is under statistical control (see Barlow and Irony 1993).

For longitudinal observational data, non-trivial sufficient statistics exist only in the class of time-transformed exponential distributions (Barlow and Proschan 1988), which is considered in this paper. This subclass of exponential family covers many distributions serving as lifetime distributions in reliability models. The exponential, Weibull, Gompertz and Pareto distributions are some of the important members of this family.

The family of time-transformed exponential distributions was considered by Spizzichino (1993) in context of the optimal design of life-testing and burn-in. However the explicit solution of the problem considered is not derived in his paper, but only some monotonicity properties of a cost function and of the observed process are given, which may be of considerable help for obtaining relevant information on a stopping region. This family was also considered by Runggaldier (1993), who developed numerical approach to finding approximate solution of burn-in problem based on stochastic control concepts using a subset of Weibull family of distributions as an example.

In this paper we derive the explicit solution of the burn-in problem. The result obtained generalizes the result of Costantini and Spizzichino (1997), who considered the burn-in problem in the case of conditionally exponential life-times. We follow closely the approach of Costantini and Spizzichino (1997). Namely, the problem considered is reformulated to an optimal stopping problem for a suitable defined Markov process and it is reduced to a free-boundary problem. In our, more general case, we have to consider tree-dimensional homogeneous strong Markov process. In the paper of Costantini and Spizzichino (1997) it is sufficient to consider, as a result of the lack of memory of the exponential distribution, a two-dimensional Markov process. To obtain the explicit solution of the problem considered, we use a different description of the evolution of observation than in the paper of Costantini and Spizzichino (1997). Our choice enables us to study the suitable monotonicity properties of some auxiliary functions in a simplified way and to obtain a simpler form of the stopping region.

This paper is organized as follows. In Sect. 2 we describe the model and the problem considered is reformulated as the optimal stopping problem for a Markov process. 
In Sect. 3 the optimal stopping problem is solved using the free-boundary method. Section 4 contains a numerical example. At the last Sect. 5 we discuss some aspects of the obtained results.

\section{Model formulation and preliminaries}

We observe $n$ identical components lifetimes with $T_{1}, T_{2}, \ldots, T_{n}$ that are conditionally i.i.d. random variables given $\Lambda=\lambda$, where $\Lambda$ is a random variable with a known distribution $\pi_{0}$. We assume that the conditional distribution of the lifetimes is given by (3). Thus the joint probability density of the vector $\left(T_{1}, \ldots, T_{n}\right)$ on the positive orthant is given by

$$
f_{n}\left(t_{1}, \ldots, t_{n}\right)=\int_{0}^{\infty} \lambda^{n}\left(\prod_{i=1}^{n} s^{\prime}\left(t_{i}\right)\right) \exp \left\{-\lambda \sum_{i=1}^{n} s\left(t_{i}\right)\right\} d \pi_{0}(\lambda) .
$$

The distribution $\pi_{0}$ can be seen as a prior distribution for an unknown parameter $\Lambda$. We assume that $\pi_{0}$ has finite $n$th moment, is not degenerate, and that $\pi_{0}([0, \lambda])>0$ for each $\lambda>0$.

The conditional failure rate function of the lifetimes is of the form

$$
r(t \mid \Lambda=\lambda)=\lambda s^{\prime}(t) \mathbf{1}_{(0, \infty)}(t)
$$

and it is non-increasing if the function $s$ is concave.

Lemma 1 If the function $s$ is concave, the unconditional distribution of $T_{1}, \ldots, T_{n}$ has one-dimensional marginals with decreasing failure rate.

Proof It is a well known fact that in the exponential case, i.e. when $s(t)=t$, the failure rate $r_{0}(t)$ is a decreasing function. In the general case considered, it can be shown that the failure rate $r(t)$ is equal to

$$
r(t)=s^{\prime}(t) r_{0}(s(t))
$$

Because $s^{\prime \prime}(t) \leq 0, r_{0}(t) \geq 0$ and $r_{0}^{\prime}(t)<0$, we have

$$
r^{\prime}(t)=s^{\prime \prime}(t) r_{0}(s(t))+\left(s^{\prime}(t)\right)^{2} r_{0}^{\prime}(s(t))<0,
$$

which completes the proof.

From Lemma 1 we have that under the assumptions concerning the conditional distribution of the lifetimes, the burn-in experiment is meaningful (Clarotti and Spizzichino 1990). Let

$$
K_{t}=n-H_{t},
$$

$t \in[0, \infty)$, be the number of components still working at time $t$, where $H_{t}$ is given by (1), and let 


$$
Y_{t}=\sum_{i=1}^{n-K_{t}} s\left(T_{(i)}\right)+s(t) K_{t}
$$

$t \in[0, \infty)$. For every $t>0,\left(Y_{t}, K_{t}\right)$ is a sufficient statistic of $\left(K_{t}, T_{(1)}, \ldots, T_{\left(n-K_{t}\right)}\right)$ for $\Lambda$, i.e. the conditional distribution of $\Lambda$ given $\mathscr{F}_{t}$ coincides with the conditional distribution of $\Lambda$ given $\left(Y_{t}, K_{t}\right)$ (Barlow and Proschan 1988). In the case of conditionally independent exponential lifetimes, i.e. $s(t)=t$, it has been shown in the paper of Costantini and Spizzichino (1997) that $(Y, K):=\left\{\left(Y_{t}, K_{t}\right)\right\}_{t \geq 0}$, is a homogeneous Markov process and that the burn-in problem reduces to an optimal stopping problem for $(Y, K)$. In this case $J(\sigma)=\mathrm{E}\left(g\left(Y_{\sigma}, K_{\sigma}\right)\right)$ for some function $g$. If $s(t) \neq t$, then the process $(Y, K)$, is not homogeneous. Moreover, $J(\sigma)$ is the expectation of a function of $Y_{\sigma}, K_{\sigma}$ and $\sigma$, what will be shown in Lemma 3. Denote

$$
U_{t}:=\sum_{i=1}^{H_{t}} s\left(T_{(i)}\right)=Y_{t}-s(t)\left(n-H_{t}\right)
$$

For every $t>0,\left(U_{t}, H_{t}, t\right)$ is a sufficient statistic of $\left(H_{t}, T_{(1)}, \ldots, T_{\left(H_{t}\right)}\right)$ for $\Lambda$, i.e. the conditional distribution of $\Lambda$ given $\mathscr{F}_{t}$ coincides with the conditional distribution of $\Lambda$ given $\left(U_{t}, H_{t}, t\right)$. We show in this section that, under (4), $(U, H, t):=$ $\left\{\left(U_{t}, H_{t}, t\right)\right\}_{t \geq 0}$ is a homogeneous Markov process and that the burn-in problem considered reduces to an optimal stopping problem for $(U, H, t)$. To apply the theory of optimal stopping of continuous time Markov processes to solving the burn-in problem considered, the life-testing experiment can be described by the homogeneous process $(Y, K, t):=\left\{\left(Y_{t}, K_{t}, t\right)\right\}_{t \geq 0}$. We use the process $(U, H, t)$ instead of the process $(Y, K, t)$ to describe the evolution of observation because $\left\{U_{t}\right\}_{t \geq 0}$ is a pure jump process, whereas $\left\{Y_{t}\right\}_{t \geq 0}$ has continuous trajectories. Our choice enables us to study the suitable monotonicity properties of some auxiliary functions in a simplified way and to obtain a simpler form of the stopping region.

Denote $\mathrm{E}=[0, \infty) \times\{0,1, \ldots, n\} \times[0, \infty)$ the state space of the process $(U, H, t)$. The family of probability measures $\left\{\pi_{u, h, t}\right\}$,

$$
d \pi_{u, h, t}(\lambda) \propto \lambda^{h} \exp \{-\lambda(u+(n-h) s(t))\} d \pi_{0}(\lambda)
$$

is the family of the conditional laws of $\Lambda$ given $\left(U_{t}, H_{t}, t\right)=(u, h, t)$. Denote

$$
p(u, h, t):=(n-h) s^{\prime}(t) \hat{\lambda}(u, h, t)
$$

where

$$
\hat{\lambda}(u, h, t):=\int_{0}^{\infty} \lambda d \pi_{u, h, t}(\lambda)=\mathrm{E}\left[\Lambda \mid\left(U_{t}, H_{t}, t\right)=(u, h, t)\right] .
$$

Lemma 2 The process $(U, H, t)$ has the strong Markov property and its infinitesimal operator for $f \in \mathscr{D}_{\mathscr{A}}=\left\{f: f(\cdot, n, t), f(u, n, \cdot) \in \mathscr{C}_{b}, f(u, h, \cdot) \in \mathscr{C}_{b}^{1}\right\}$ is of the form 


$$
\mathscr{A} f(u, h, t)= \begin{cases}p(u, h, t)[f(u+s(t), h+1, t)-f(u, h, t)] & \\ +\frac{\partial f}{\partial t}(u, h, t), & \text { for } h<n, \\ 0, & \text { for } h=n,\end{cases}
$$

where the function $p(u, h, t)$ is given by (7).

Proof We begin by the analysis of the process $\left\{H_{t}\right\}_{t \geq 0}$. It is a counting process with $\left\{\mathscr{F}_{t} \vee \sigma(\Lambda)\right\}$-intensity $\left\{\left(n-H_{t}\right) s^{\prime}(t) \Lambda\right\}_{t \geq 0}$ and hence (Bremaud 1981, Theorem T14) with $\left\{\mathscr{F}_{t}\right\}$-intensity $\left\{p\left(U_{t}, H_{t}, t\right)\right\}_{t \geq 0}$, where $p(u, h, t)$ is given by (7). By the DoobMeyer decomposition

$$
d H_{t}=p\left(U_{t}, H_{t}, t\right) d t+d M_{t},
$$

where $M_{t}$ is a uniquely determined martingale. It follows that for every bounded measurable $f$

$$
\begin{aligned}
M_{t}^{f}:= & \int_{0}^{t}\left[f\left(U_{u-}+s(u), H_{u-}+1, u-\right)-f\left(U_{u-}, H_{u-}, u-\right)\right] d M_{u} \\
= & \sum_{u \leq t}\left[f\left(U_{u}, H_{u}, u\right)-f\left(U_{u-}, H_{u-}, u-\right)\right]-\int_{0}^{t}\left[f\left(U_{u}+s(u), H_{u}+1, u\right)\right. \\
& \left.-f\left(U_{u}, H_{u}, u\right)\right] p\left(U_{u}, H_{u}, u\right) d u
\end{aligned}
$$

is also a martingale for every bounded, measurable $f$.

On the other hand, from Ito's Lemma

$$
\begin{aligned}
f\left(U_{t}, H_{t}, t\right)= & f(0,0,0)+\int_{0}^{t} \frac{\partial f}{\partial t}\left(U_{u}, H_{u}, u\right) d u \\
& +\sum_{u \leq t}\left[f\left(U_{u}, H_{u}, u\right)-f\left(U_{u-}, H_{u-}, u-\right)\right] .
\end{aligned}
$$

Combining (10) with (11) and applying notation (9), we get

$$
\begin{aligned}
f\left(U_{t}, H_{t}, t\right)= & f(0,0,0)+\int_{0}^{t}\left[f\left(U_{u}+s(u), H_{u}+1, u\right)-f\left(U_{u}, H_{u}, u\right)\right] \\
& \times\left(n-H_{u}\right) s^{\prime}(u) \hat{\lambda}\left(U_{u}, H_{u}, u\right) d u+\int_{0}^{t} \frac{\partial f}{\partial t}\left(U_{u}, H_{u}, u\right) d u+M_{t}^{f} \\
= & f(0,0,0)+M_{t}^{f}+\int_{0}^{t} \mathscr{A}\left(U_{u}, H_{u}, u\right) d u .
\end{aligned}
$$


Thus, it is easy to see, that the equation above describes a martingale problem, whose solution has the strong Markov property, and the infinitesimal operator $\mathscr{A}$ of the process $(U, H, t)$ is of the form (9).

For every $w \in L^{1}\left(\pi_{0}\right)$, let

$$
\hat{w}(u, h, t)=\int_{0}^{\infty} w(\lambda, t) d \pi_{u, h, t}(\lambda)=\mathrm{E}\left[w(\Lambda, t) \mid\left(U_{t}, H_{t}, t\right)=(u, h, t)\right]
$$

We will denote by

$$
\rho(\lambda, t):=\mathrm{E}[C(T-t) \mid \Lambda=\lambda, T>t]
$$

where for a given $\lambda$, the density of $T$ is given by (3).

Lemma 3 For every stopping time $\sigma \in \mathscr{T}$, the expected loss function given by (2) can be represented as

$$
J(\sigma)=\mathrm{E}\left[g\left(U_{\sigma}, H_{\sigma}, \sigma\right)\right]
$$

where

$$
g(u, h, t):=c h+(n-h) \hat{\rho}(u, h, t),
$$

and $\hat{\rho}$ is defined by (13) and (12). Moreover, for all $(u, h, t) \in \mathrm{E}$

$$
\mathscr{A} g(u, h, t)=p(u, h, t)[c-\hat{\gamma}(u+s(t), h+1, t)],
$$

where $\hat{\gamma}$ is defined by (12) and

$$
\gamma(\lambda, t):=\rho(\lambda, t)-\frac{\frac{\partial \rho}{\partial t}(\lambda, t)}{s^{\prime}(t) \lambda}
$$

and $p(u, h, t)$ is given by (7).

Proof For every $\sigma \in \mathscr{T}$, we have

$$
\begin{aligned}
J(\sigma) & =\mathrm{E}\left[c H_{\sigma}+\mathbf{1}_{\left\{H_{\sigma}<n\right\}} \sum_{j=H_{\sigma}+1}^{n} C\left(T_{(j)}-\sigma\right)\right] \\
& =\mathrm{E}\left[\mathrm{E}\left[c H_{\sigma}+\mathbf{1}_{\left\{H_{\sigma}<n\right\}} \sum_{j=H_{\sigma}+1}^{n} C\left(T_{(j)}-\sigma\right) \mid \mathscr{F}_{\sigma}\right]\right]
\end{aligned}
$$


and

$$
\mathrm{E}\left[\sum_{j=H_{\sigma}+1}^{n} C\left(T_{(j)}-\sigma\right) \mid \mathscr{F}_{\sigma}\right]=\mathrm{E}\left[\int_{\sigma^{+}}^{+\infty} C(r-\sigma) d K(r) \mid \mathscr{F}_{\sigma}\right] .
$$

Since the process $(U, H, t)$ has the strong Markov property, and

$$
\begin{aligned}
\mathrm{E}\left[\sum_{j=H_{t}+1}^{n} C\left(T_{(j)}-t\right) \mid \mathscr{F}_{t}\right] & =\mathrm{E}\left[\sum_{j=H_{t}+1}^{n} C\left(T_{(j)}-t\right) \mid\left(U_{t}, H_{t}, t\right)\right] \\
& =\left(n-H_{t}\right) \mathrm{E}\left[\mathrm{E}[C(T-t) \mid \Lambda, T>t] \mid\left(U_{t}, H_{t}, t\right)\right]
\end{aligned}
$$

and applying notation (13) and (14), we have

$$
J(\sigma)=\mathrm{E}\left[c H_{\sigma}+\left(n-H_{\sigma}\right) \hat{\rho}\left(U_{\sigma}, H_{\sigma}, \sigma\right)\right]=\mathrm{E}\left[g\left(U_{\sigma}, H_{\sigma}, \sigma\right)\right] .
$$

By Lemma 2

$$
\begin{aligned}
\mathscr{A} g(u, h, t)= & p(u, h, t)[g(u+s(t), h+1, t)-g(u, h, t)]+\frac{\partial g}{\partial t}(u, h, t) \\
& +p(u, h, t)[c+(n-h-1) \hat{\rho}(u+s(t), h+1, t)-(n-h) \hat{\rho}(u, h, t)] \\
& +(n-h) \frac{\partial \hat{\rho}}{\partial t}(u, h, t) .
\end{aligned}
$$

But

$$
\frac{\partial \hat{\rho}}{\partial t}(u, h, t)=\frac{\widehat{\partial \rho}}{\partial t}(u, h, t)+p(u, h, t)[\hat{\rho}(u, h, t)-\hat{\rho}(u+s(t), h+1, t)],
$$

therefore

$$
\mathscr{A} g(u, h, t)=p(u, h, t)\left[c-\rho(u+s(t), h+1, t)+\frac{\widehat{\frac{\partial \rho}{\partial t}}(u, h, t)}{s^{\prime}(t) \hat{\lambda}(u, h, t)}\right] .
$$

Notice, that

$$
\frac{\frac{\partial \rho}{\partial t}(u, h, t)}{\hat{\lambda}(u, h, t)}=\frac{\widehat{\partial \rho}}{\partial t} \frac{1}{\lambda}(u+s(t), h+1, t) .
$$

After applying the result above and notation (16), we obtain formula (15), and the proof is complete. 


\section{Solution of the burn-in optimal stopping problem}

By Lemmas 2 and 3, the burn-in problem considered reduces to the optimal stopping problem for the Markov process $(U, H, t)$, namely, to finding $\sigma^{*} \in \mathscr{T}$ such that

$$
\mathrm{E}\left[g\left(U_{\sigma^{*}}, H_{\sigma^{*}}, \sigma^{*}\right)\right]=\inf _{\sigma \in \mathscr{T}} \mathrm{E}\left[g\left(U_{\sigma}, H_{\sigma}, \sigma\right)\right]
$$

Under the assumptions concerning the function $C$

$$
\mathrm{E}\left[\sup _{\sigma \in \mathscr{T}}\left|g\left(U_{t}, H_{t}, t\right)\right|\right]<\infty,
$$

therefore such the optimal stopping time $\sigma^{*}$ exists in our problem.

By the general theory of optimal stopping of continuous-time Markov processes (see Peskir and Shiryaev 2006), the optimal stopping time

$$
\sigma^{*}=\inf \left\{t \geq 0:\left(U_{t}, H_{t}, t\right) \in \Gamma\right\},
$$

where

$$
\Gamma=\{(u, h, t): g(u, h, t)=v(u, h, t)\},
$$

and

$$
v(u, h, t)=\inf _{\sigma \in \mathscr{T}, \sigma \geq t} \mathrm{E}\left[g\left(U_{\sigma}, H_{\sigma}, \sigma\right) \mid U_{t}=u, H_{t}=h\right]
$$

Consider the free-boundary problem

$$
\begin{aligned}
& g^{*}(u, h, t)=g(u, h, t), \quad \text { when }(u, h, t) \in \Gamma^{*}, \\
& \mathscr{A} g^{*}(u, h, t)=0, \quad \text { when }(u, h, t) \in \bar{\Gamma}^{*} \text {, }
\end{aligned}
$$

where $\bar{\Gamma}^{*}$ denotes the complement of $\Gamma^{*}$ in E. It follows from the regular characterization of the value function (see Peskir and Shiryaev 2006) that if there exists a solution $\left(\Gamma^{*}, g^{*}\right)$ of (17) that is smooth enough, and that also satisfies

$$
\begin{aligned}
g^{*}(u, h, t) & <g(u, h, t), & \text { when }(u, h, t) & \in \bar{\Gamma}^{*}, \\
\mathscr{A} g^{*}(u, h, t) & \geq 0, & \text { when }(u, h, t) & \in \Gamma^{*},
\end{aligned}
$$

then this solution must be $(\Gamma, v)$.

To solve the burn-in problem we should find the form of the set $\Gamma$ or the form of the value function $v$. The following lemma will be useful for determining the monotonicity properties of a function which will be used to describe a solution of the problem considered. 
Lemma 4 If the function $w(\lambda, t)$ is an increasing function of $\lambda$ for each $t$ and a nonincreasing function of t for each $\lambda$, then the function $\hat{w}(u, h, t)$ is a decreasing function of $u$ for each $h$ and $t$, an increasing function of $h$ for each $u$ and $t$, and a decreasing function of $t$ for each $u$ and $h$.

Proof Let us first prove that $\hat{w}(u, h, t)$ is a decreasing function of $u$. For fixed $h$ and $t$, the family $d \pi_{u, h, t}(\lambda)$ parametrized by $u$, has a monotone likelihood ratio in $-\lambda$. Thus for any increasing function $\Psi(\lambda), \int_{0}^{\infty} \Psi(\lambda) d \pi_{u, h, t}(\lambda)$ is a decreasing function of $u$. It immediately follows, that $\hat{w}(u, h, t)$ is a decreasing function of $u$.

Using the same reasoning and a reparametrization of the family $d \pi_{u, h, t}(\lambda)$ as $d \pi_{y, h}^{\prime}(\lambda) \propto \lambda^{h} e^{-\lambda y} \pi_{0}(\lambda)$ with $y=u+(n-h) s(t)$, it can be shown that

$$
\mathrm{E}\left[w(\Lambda, t) \mid Y_{t}=y, H_{t}=h\right]<\mathrm{E}\left[w(\Lambda, t) \mid Y_{t}=y, H_{t}=h+1\right],
$$

and equivalently

$$
\hat{w}(u, h, t)<\hat{w}(u+s(t), h+1, t) .
$$

Because $\hat{w}(u, h, t)$ is a decreasing function of $u$, we have $\hat{w}(u, h, t)<\hat{w}(u, h+1, t)$. It remains to show, that $\hat{w}(u, h, t)$ is a decreasing function of $t$. We have

$$
\frac{\partial \hat{w}}{\partial t}(u, h, t)=\frac{\widehat{\partial w}}{\partial t}(u, h, t)+p(u, h, t)[\hat{w}(u, h, t)-\hat{w}(u+s(t), h+1, t)],
$$

where $p(u, h, t)$ is given by (7). By inequality (19) and the fact, that $p(u, h, t)>0$ for each $t$, we have

$$
\frac{\partial \hat{w}}{\partial t}(u, h, t)<\frac{\widehat{\partial w}}{\partial t}(u, h, t) .
$$

By the assumption that $w(\lambda, t)$ is a non-increasing function of $t$, we obtain inequality $\frac{\partial w}{\partial t}(\lambda, t) \leq 0$, which implies that both $\frac{\widehat{\partial w}}{\partial t}(u, h, t)$ and $\frac{\partial \hat{w}}{\partial t}(u, h, t)<0$, and completes the proof.

Corollary 1 The function $p(u, h, t)$ given by (7) is a decreasing function of $u$ for each $h$ and $t$.

Corollary 2 Assume, that the function $\gamma(\lambda, t)$ given by (16) satisfies the following conditions

(i) it is an increasing function of $\lambda$ and a non-increasing function of $t$,

(ii) $\lim _{t \rightarrow \infty} \hat{\gamma}(s(t), n, t)<c$.

Then $\hat{\gamma}(u, h, t)$ is a decreasing function of $u$ for each $h$ and $t$, an increasing function of $h$ for each $u$ and $t$, and a decreasing function of $t$ for each $u$ and $h$. Moreover for each $h$ and $u$,

$$
\lim _{t \rightarrow \infty} \hat{\gamma}(u+s(t), h+1, t)<c .
$$


Remark 1 The assumptions of Corollary 2 are usually easy to verify in specific cases (see Sect. 4 for example). If the assumptions concerning the cost function $C$ are satisfied, the function $s$ is concave and $\frac{s^{\prime \prime}(t)}{s^{\prime}(t)}$ is a non-decreasing function of $t$, then the function $\gamma(\lambda, t)$ satisfies the assumption $(i)$ of Corollary 2.

Motivated by the monotonicity properties of the function $\hat{\gamma}(u, h, t)$, given in Corollary 2 , and formula (15), we suggest that the set $\Gamma$ is of the form

$$
\Gamma=\Gamma_{0} \cup \Gamma_{1} \cup \cdots \cup \Gamma_{n},
$$

where $\Gamma_{h}=\left\{(u, h, t): t \geq t_{h}(u), u \geq 0\right\}$ for $h \leq n$, and $t_{n}(u)=0$. Furthermore, the function $v$ satisfies the following conditions.

Lemma 5 For $(u, h, t) \in \Gamma$

$$
\mathscr{A} v(u, h, t)=p(u, h, t) q(u, h, t),
$$

where

$$
q(u, h, t):=c-\hat{\gamma}(u+s(t), h+1, t)+(v-g)(u+s(t), h+1, t),
$$

and $p(u, h, t)$ is given by (7). For $(u, h, t) \in \bar{\Gamma}$

$$
\frac{\partial(v-g)}{\partial t}(u, h, t)=p(u, h, t)[(v-g)(u, h, t)-q(u, h, t)] .
$$

Proof By Lemma 2 and the fact, that $v(u, h, t)=g(u, h, t)$ for $(u, h, t) \in \Gamma$, we obtain

$$
\begin{aligned}
\mathscr{A} v(u, h, t) & =p(u, h, t)[v(u+s(t), h+1, t)-v(u, h, t)]+\frac{\partial v}{\partial t}(u, h, t) \\
& =p(u, h, t)[v(u+s(t), h+1, t)-g(u, h, t)]+\frac{\partial g}{\partial t}(u, h, t) \\
& =\mathscr{A} g(u, h, t)+p(u, h, t)(v-g)(u+s(t), h+1, t) .
\end{aligned}
$$

Using formula (15)

$$
\begin{aligned}
\mathscr{A} v(u, h, t) & =p(u, h, t)[c-\hat{\gamma}(u+s(t), h+1, t)+(v-g)(u+s(t), h+1, t)] \\
& =p(u, h, t) q(u, h, t),
\end{aligned}
$$

which completes the first part of the lemma.

Applying formula (9) to the function $v-g$ we have

$$
\begin{aligned}
\mathscr{A}(v-g)(u, h, t)= & p(u, h, t)[(v-g)(u+s(t), h+1, t)-(v-g)(u, h, t)] \\
& +\frac{\partial(v-g)}{\partial t}(u, h, t) .
\end{aligned}
$$


From the linearity of the operator $\mathscr{A}$, and the fact that $\mathscr{A} v(u, h, t)=0$ for $(u, h, t) \in$ $\bar{\Gamma}$, we get

$$
\mathscr{A}(v-g)(u, h, t)=-\mathscr{A} g(u, h, t)=p(u, h, t)[c-\hat{\gamma}(u+s(t), h+1, t)] .
$$

Comparing the right hand sides of Eqs. (23) and (24), and using notation (21), we obtain formula (22), which completes the proof.

Theorem 1 Assume that the conditions of Corollary 2 hold. Then, the solution $v$ to the free-boundary problem given by (17) and (18) can be derived recursively from the following equations

$$
v(u, n, t)=g(u, n, t)
$$

for all $u \geq 0$ and $t \geq 0$, and for $h<n$

$$
v(u, h, t)= \begin{cases}g(u, h, t), & \text { for } t \geq t_{h}(u), \\ g(u, h, t)+\int_{t}^{t_{h}(u)} p(u, h, z) q(u, h, z) & \\ \times \exp \left\{-\int_{t}^{z} p(u, h, x) d x\right\} d z, & \text { for } t<t_{h}(u),\end{cases}
$$

where for a given $h$ and $u, t_{h}(u)$ is the unique solution to the equation

$$
q(u, h, t)=0
$$

or $t_{h}(u)=0$, if Eq. (26) has no solution in $[0, \infty)$, where the function $p$ and $q$ are given by (7) and (21), respectively.

Proof The proof will be carried out by backward induction with respect to $h$. For $h=n$ we have $v(u, n, t)=g(u, n, t)$ and $\mathscr{A} v(u, n, t)=0$ (by Lemma 2) for all $u \geq 0, t \geq$ 0 . Therefore the conditions (17) and (18) are satisfied with

$$
\Gamma_{n}=\{(u, n, t): u \geq 0, t \geq 0\}
$$

For $h=n-1$, we have $v(u, n-1, t)=g(u, n-1, t)$, when $t \geq t_{n-1}(u)$, and

$$
\begin{aligned}
v(u, n-1, t)= & g(u, n-1, t)+\int_{t}^{t_{n-1}(u)} p(u, n-1, z) q(u, n-1, z) \\
& \times \exp \left\{-\int_{t}^{z} p(u, n-1, x) d x\right\} d z,
\end{aligned}
$$


when $t<t_{n-1}(u)$, where $p(u, n-1, t)$ is given by (7) and

$$
q(u, n-1, t)=c-\hat{\gamma}(u+s(t), n, t) .
$$

By the monotonicity properties of the function $\hat{\gamma}$, given in Corollary 2 , we have that the function $q(u, n-1, t)$ is an increasing function of $u$ for each $t$, and an increasing function of $t$ for each $u$. Moreover, for every given $u$, it changes sign from negative to positive exactly once at the point $t_{n-1}(u)$ or it is positive for all $t$, and then we put $t_{n-1}(u)=0$. Therefore, for each $u \geq 0$

$$
\begin{aligned}
& v(u, n-1, t)=g(u, n-1, t), \quad \text { when } t \geq t_{n-1}(u), \\
& v(u, n-1, t)<g(u, n-1, t), \quad \text { when } t<t_{n-1}(u),
\end{aligned}
$$

because $p(u, h, t)>0$ for all $u, h$ and $t$. By Lemma 5 the function $v$ also satisfies the conditions imposed on the infinitesimal operator $\mathscr{A} v(u, n-1, t)$. Namely, $\mathscr{A} v(u, n-1, t) \geq 0$, when $(u, n-1, t) \in \Gamma_{n-1}=\left\{(u, n-1, t): t \geq t_{n-1}(u)\right\}$. Moreover, the function $v$ is a solution to the differential equation (22), which was derived under the condition $\mathscr{A} v(u, h, t)=0$ for $(u, h, t) \in \bar{\Gamma}_{h}$. Therefore $\mathscr{A} v(u, n-1, t)=$ 0 , when $(u, n-1, t) \in \bar{\Gamma}_{n-1}$.

Let us assume that for a given $h$ the function $q(u, h, t)$, given by (21), is an increasing function of $u$ for each $t$, and an increasing function of $t$ for each $u$. Therefore, by Corollary 2 , for every given $u$ it changes sign from negative to positive exactly once at the point $t_{h}(u)$ or it is positive for all $t$, and then we put $t_{h}(u)=0$. These monotonicity properties suffice to show, in the analogous way as for $h=n-1$, that the function $v(u, h, t)$ satisfies the conditions (17) and (18).

We show that $q(u, h-1, t)$ has the same monotonicity properties as $q(u, h, t)$.

First we show that

$$
(v-g)(u, h, t)= \begin{cases}\int_{t}^{t_{h}(u)} p(u, h, z) q(u, h, z) \exp \left\{-\int_{t}^{z} p(u, h, x) d x\right\} d z, & \text { for } t<t_{h}(u), \\ 0, & \text { for } t \geq t_{h}(u)\end{cases}
$$

is an increasing function of $u$ for each $t$, where $t_{h}(u)$ is the unique solution to Eq. (26) or $t_{h}(u)=0$, if Eq. (26) has no solution. Let us note that

$$
f_{u h t}(z):= \begin{cases}0, & \text { for } z \leq t \\ p(u, h, z) \exp \left\{-\int_{t}^{z} p(u, h, x) d x\right\}, & \text { for } z>t\end{cases}
$$

is a density function of a random variable, say $Z_{u h t}$, with a reliability function

$$
\bar{F}_{u h t}(z)=\exp \left\{-\int_{t}^{z} p(u, h, x) d x\right\} \mathbf{1}_{(t, \infty)}(z)
$$

Let $u_{1}<u_{2}$ and $Z_{u_{1} h t}, Z_{u_{2} h t}$ be the random variables with density functions $f_{u_{1} h t}, f_{u_{2} h t}$, respectively. By Corollary $1, \bar{F}_{u_{1} h t}(z) \leq \bar{F}_{u_{2} h t}(z)$ what implies 
$Z_{u_{1} h t} \leq^{s t} Z_{u_{2} h t}$. From the assumption that $q(u, h, t)$ is an increasing function of $u$ we have $t_{h}\left(u_{1}\right)<t_{h}\left(u_{2}\right)$ or $t_{h}\left(u_{2}\right)=0$. In the first case

$$
\begin{aligned}
(v-g)\left(u_{1}, h, t\right) & =\int_{-\infty}^{t_{h}\left(u_{1}\right)} q\left(u_{1}, h, z\right) f_{u_{1} h t}(z) d z \\
& <\int_{-\infty}^{t_{h}\left(u_{2}\right)} q\left(u_{2}, h, z\right) f_{u_{1} h t}(z) d z=\operatorname{E} \phi\left(Z_{u_{1} h t}\right),
\end{aligned}
$$

where

$$
\phi(z):=q\left(u_{2}, h, z\right) \mathbf{1}_{\left(\infty, t_{h}\left(u_{2}\right)\right)}(z)
$$

is an increasing function of $z$ from the inductive assumption that $q(u, h, t)$ is an increasing function of $t$ for each $u$. Since that $Z_{u_{1} h t} \leq^{s t} Z_{u_{2} h t}$ we have

$$
\mathrm{E} \psi\left(Z_{u_{1} h t}\right) \leq \mathrm{E} \psi\left(Z_{u_{2} h t}\right)
$$

for each non-decreasing function $\psi$ such that the above expectations exist. In the special case $\psi=\phi$

$$
(v-g)\left(u_{1}, h, t\right)<\mathrm{E} \phi\left(Z_{u_{1} h t}\right) \leq \mathrm{E} \phi\left(Z_{u_{2} h t}\right)=(v-g)\left(u_{2}, h, t\right) .
$$

In the case when $t_{h}\left(u_{2}\right)=0$ we have $(v-g)\left(u_{2}, h, t\right)=0$ for each $t$ and therefore $(v-g)\left(u_{1}, h, t\right)<(v-g)\left(u_{2}, h, t\right)$ trivially. Therefore, $(v-g)(u, h, t)$ is an increasing function of $u$ for each $t$.

Now we show that $(v-g)(u, h, t)$ is an increasing function of $t$ for each given $u$. Let $G(u, h, t)$ be a function for which

$$
\frac{\partial}{\partial t} G(u, h, t)=p(u, h, t)
$$

The function

$$
\begin{aligned}
(v-g)(u, h, t) & =\exp \{G(u, h, t)\} \int_{t}^{t_{h}(u)} p(u, h, z) q(u, h, z) \exp \{-G(u, h, z)\} d z \\
& =-\exp \{G(u, h, t)\} \int_{t}^{t_{h}(u)} q(u, h, z) \frac{\partial}{\partial z} \exp \{-G(u, h, z)\} d z .
\end{aligned}
$$


Applying integration by parts, we have

$$
(v-g)(u, h, t)=q(u, h, t)+\exp \{G(u, h, t)\} \int_{t}^{t_{h}(u)} \frac{\partial}{\partial z} q(u, h, z) \exp \{-G(u, h, z)\} d z .
$$

Therefore

$$
\begin{aligned}
\frac{\partial}{\partial t}(v-g)(u, h, t)= & \exp \{G(u, h, t)\} p(u, h, t) \int_{t}^{t_{h}(u)} \frac{\partial}{\partial z} q(u, h, z) \\
& \times \exp \{-G(u, h, z)\} d z,
\end{aligned}
$$

and from the assumption that $\frac{\partial}{\partial t} q(u, h, t)>0$ we have $\frac{\partial}{\partial t}(v-g)(u, h, t)>0$. We have showed that $(v-g)(u, h, t)$ is an increasing function of $u$ for each $t$, and an increasing function of $t$ for each $u$. Therefore, by Corollary 2 and the monotonicity properties of the function $(v-g)(u, h, t)$,

$$
q(u, h-1, t)=c-\hat{\gamma}(u+s(t), h, t)+(v-g)(u+s(t), h, t)
$$

is an increasing function of $u$ for each $t$, an increasing function of $t$ for each $u$, and for every given $u$ it changes sign from negative to positive exactly once at the point $t_{h-1}(u)$ or it is positive for all $t$, and then we put $t_{h-1}(u)=0$.

To complete the proof we need to show that $v \in \mathscr{D} \mathscr{A}$. By the form (25) of the function $v$ and since $g \in \mathscr{D} \mathscr{A}$ it suffices to show that the function $v(u, h, \cdot)$ is differentiable at the points $t_{h}(u)$ for $h=0,1, \cdots, n-1$ and $u \in[0, \infty)$. But it is easily seen from the formula (27).

Corollary 3 The optimal burn-in time is of the form

$$
\sigma^{*}=\inf \left\{t: t \geq t_{h}(u), \text { where } h=H_{t}, u=U_{t}\right\},
$$

where $t_{h}(u)$ is the solution to Eq. (26) or $t_{h}(u)=0$, if Eq. (26) has no solution.

Remark 2 From the fact that, under the conditions of Corollary 2, for a given $h$ and $t$ the function $q(u, h, t)$ is an increasing function of $u$, for all $h \in\{0,1, \ldots, n-1\}$, if $u_{1}<u_{2}$, then $t_{h}\left(u_{1}\right)>t_{h}\left(u_{2}\right)$, when $t_{h}\left(u_{1}\right)>0$ or $t_{h}\left(u_{1}\right)=t_{h}\left(u_{2}\right)=0$, otherwise.

Remark 3 From the fact that, under the conditions of Corollary 2, the function $\hat{\gamma}(u, h, t)$ is an increasing function of $h$ for each $u$ and $t$, and $q(u, h, t)$ is an increasing function of $t$ for each $u$ and $h$, for all $h \in\{0,1, \ldots, n-2\}$, and for all $u \geq 0$

$$
\begin{aligned}
q\left(u, h, t_{h+1}(u)\right)= & c-\hat{\gamma}\left(u+s\left(t_{h+1}(u)\right), h+1, t_{h+1}(u)\right) \\
> & c-\hat{\gamma}\left(u+s\left(t_{h+1}(u)\right), h+2, t_{h+1}(u)\right) \\
\geq & c-\hat{\gamma}\left(u+s\left(t_{h+1}(u)\right), h+2, t_{h+1}(u)\right) \\
& +(v-g)\left(u+s\left(t_{h+1}(u)\right), h+2, t_{h+1}(u)\right) \\
= & q\left(u, h+1, t_{h+1}(u)\right)=0,
\end{aligned}
$$


which implies $t_{h}(u)<t_{h+1}(u)$, when $t_{h+1}(u)>0$ or $t_{h}(u)=t_{h+1}(u)=0$, otherwise.

Remark 4 Using the process $(Y, H, t)$ instead of the process $(U, H, t)$ for a description of the evolution of observation would lead to the stopping region given by a sequence of functions, say $v_{h}(y)$, of the argument $y$ instead of the functions $t_{h}(u)$ of the argument $u$. The optimal burn-in time would be of the form

$$
\tau^{*}=\inf \left\{t: t \geq v_{h}(y), \text { where } h=H_{t}, y=Y_{t}\right\} .
$$

Because of the process $Y_{t}, t \geq 0$, has continuous trajectories, this solution would be more difficult to apply in practice. Moreover, to obtain the form of the value function a partial differential equation would have to be solved instead of the ordinary differential equation given by (22).

\section{Example}

Let us assume that the prior distribution $\pi_{0}$ of the parameter $\lambda$ is the gamma distribution $\mathscr{G}\left(\alpha_{0}, \beta_{0}\right)$ with density

$$
\Gamma\left(\alpha_{0}\right)^{-1} \beta_{0}^{\alpha_{0}} \vartheta^{\alpha_{0}-1} \exp \left(-\beta_{0} \vartheta\right) \mathbf{1}_{(0, \infty)}(\vartheta)
$$

where $\alpha_{0}, \beta_{0}>0$ are known parameters. It is easy to show that the family of gamma distributions is the conjugate one to the family of distributions given by (3), and the posterior distribution $\pi_{t}$ is the gamma distribution $\mathscr{G}\left(\alpha_{t}, \beta_{t}\right)$, where $\alpha_{t}=\alpha_{0}+h, \beta_{t}=$ $\beta_{0}+u+(n-h) s(t)$.

We further assume, that the function $s(t)$ is concave, $\frac{s^{\prime \prime}(t)}{s^{\prime}(t)}$ is a non-decreasing function of $t$ and the function $C(r)$ is of the form

$$
C(r):= \begin{cases}c_{0}, & \text { for } r \leq W, \\ c_{1}, & \text { for } r>W,\end{cases}
$$

where $W>0$ and $c_{1}<c<c_{0}$.

We have

$$
\gamma(\lambda, t)=c_{0}-\left(c_{0}-c_{1}\right) \frac{s^{\prime}(t+W)}{s^{\prime}(t)} \exp \{-\lambda(s(t+W)-s(t))\}
$$

and

$\hat{\gamma}(u, h, t)=c_{0}-\left(c_{0}-c_{1}\right) \frac{s^{\prime}(t+W)}{s^{\prime}(t)}\left(\frac{\beta_{0}+u+(n-h) s(t)}{\beta_{0}+u+(n-h-1) s(t)+s(t+W)}\right)^{\alpha_{0}+h}$.

Because $s(t)$ is an increasing function of $t$ and $c_{0}>c_{1}, \gamma(\lambda, t)$ is an increasing function of $\lambda$. From the assumption that $s(t)$ is concave, $\exp \{-\lambda(s(t+W)-s(t))\}$ is a non-decreasing function of $t$. Moreover, by assumption that $\frac{s^{\prime \prime}(t)}{s^{\prime}(t)}$ is a non-decreasing 
function, we have $\frac{s^{\prime \prime}(t+W)}{s^{\prime}(t+W)} \geq \frac{s^{\prime \prime}(t)}{s^{\prime}(t)}$ and $s^{\prime \prime}(t+W) s^{\prime}(t)-s^{\prime}(t+W) s^{\prime \prime}(t) \geq 0$. Above leads to

$$
\frac{\partial}{\partial t} \frac{s^{\prime}(t+W)}{s^{\prime}(t)}=\frac{s^{\prime \prime}(t+W) s^{\prime}(t)-s^{\prime}(t+W) s^{\prime \prime}(t)}{\left(s^{\prime}(t)\right)^{2}} \geq 0,
$$

and proves that $\gamma(\lambda, t)$ is an increasing function of $\lambda$ for each $t$ and a non-increasing function of $t$ for each $\lambda$. Finally,

$$
\lim _{t \rightarrow \infty} \hat{\gamma}(s(t), n, t)=\lim _{t \rightarrow \infty}\left[c_{0}-\left(c_{0}-c_{1}\right) \frac{s^{\prime}(t+W)}{s^{\prime}(t)}\left(\frac{\beta_{0}+s(t)}{\beta_{0}+s(t+W)}\right)^{\alpha_{0}+n}\right]<c
$$

if and only if

$$
\lim _{t \rightarrow \infty} \frac{s^{\prime}(t+W)}{s^{\prime}(t)}<\left(\frac{c_{0}-c_{1}}{c_{0}-c}\right)^{\frac{1}{\alpha_{0}+n-1}}
$$

From the assumption that $s(t)$ is concave and $\frac{s^{\prime \prime}(t)}{s^{\prime}(t)}$ is a non-decreasing function of $t, \frac{s^{\prime}(t+W)}{s^{\prime}(t)}<1$ and is non-decreasing function of $t$, and thus there exists

$$
\lim _{t \rightarrow \infty} \frac{s^{\prime}(t+W)}{s^{\prime}(t)} \leq 1
$$

On the other hand, because $c_{1}<c<c_{0},\left(\frac{c_{0}-c_{1}}{c_{0}-c}\right)^{\frac{1}{\alpha_{0}+n-1}}>1$, and conditions (28) and (29) hold.

By Corollary 2, the $\hat{\gamma}(u, h, t)$ is a decreasing function of $u$ for each $h$ and $t$, an increasing function of $h$ for each $u$ and $t$ and a decreasing function of $t$ for each $u$ and $h$. Moreover, for each $u$ and $h$,

$$
\lim _{t \rightarrow \infty} \hat{\gamma}(u+s(t), h+1, t)<c
$$

In the case considered, the assumptions concerning function $\gamma$ are satisfied. It is easy to obtain that

$$
\hat{\lambda}(u, h, t)=\frac{\alpha_{0}+h}{\beta_{0}+u+(n-h) s(t)},
$$


and

$$
(v-g)(u, h, t)= \begin{cases}0, & \text { for } t \geq t_{h}(u), \\ \left(\alpha_{0}+h\right)\left(\beta_{0}+u+(n-h) s(t)\right)^{\alpha_{0}+h} & \\ \cdot \int_{t}^{t_{h}(u)} \frac{(n-h) s^{\prime}(z) q(u, h, z)}{\left(\beta_{0}+u+(n-h) s(z)\right)^{\alpha_{0}+h+1}} d z, & \text { for } t<t_{h}(u) .\end{cases}
$$

Consider the case when $n=2$. By Theorem $1, v(u, 2, t)=g(u, 2, t)$, and $t_{2}(u)=$ 0 . To obtain $t_{1}(u)$, and consequently $v(u, 1, t)$, we have to solve the equation

$$
q(u, 1, t)=\left(c-c_{0}\right)+\left(c_{0}-c_{1}\right) \frac{s^{\prime}(t+W)}{s^{\prime}(t)}\left(\frac{\beta_{0}+u+s(t)}{\beta_{0}+u+s(t+W)}\right)^{\alpha_{0}+2}=0
$$

with respect to $t$ for each $u \geq 0$. Let $t_{1}(u)$ be the solution to Eq. (30) in $[0, \infty)$ or $t_{1}(u)=0$ if Eq. (30) has no solution in $[0, \infty)$.

The function $q(u, 0, t)$ for $t \in\left(0, t_{1}^{\prime}(u)\right)$, where $t_{1}^{\prime}(u)=\inf \left\{t: t \geq t_{1}(u+s(t))\right\}$ is of the form

$$
\begin{aligned}
q(u, 0, t)= & c-\hat{\gamma}(u+s(t), 1, t)+(v-g)(u+s(t), 1, t)=c-\hat{\gamma}(u+s(t), 1, t) \\
& +\left(\alpha_{0}+1\right)\left(\beta_{0}+u+2 s(t)\right)^{\alpha_{0}+1} \int_{t}^{t_{1}(u+s(t))} \frac{s^{\prime}(z) q(u+s(t), 1, z)}{\left(\beta_{0}+u+s(t)+s(z)\right)^{\alpha_{0}+2}} d z .
\end{aligned}
$$

But

$$
\begin{aligned}
& \left(\alpha_{0}+1\right) \int_{t}^{t_{1}(u+s(t))} \frac{s^{\prime}(z) q(u+s(t), 1, z)}{\left[\beta_{0}+u+2 s(z)\right]^{\alpha_{0}+2}} d z \\
& =\left(c-c_{0}\right)\left\{\frac{1}{\left[\beta_{0}+u+2 s(t)\right]^{\alpha_{0}+1}}-\frac{1}{\left[\beta_{0}+u+s(t)+s\left(t_{1}(u+s(t))\right)\right]^{\alpha_{0}+1}}\right\} \\
& \quad+\left(c_{0}-c_{1}\right)\left\{\frac{1}{\left[\beta_{0}+u+s(t)+s(t+W)\right]^{\alpha_{0}+1}}-\frac{1}{\left[\beta_{0}+u+s(t)+s\left(t_{1}(u+s(t))+W\right)\right]^{\alpha_{0}+1}}\right\} .
\end{aligned}
$$

Finally, for any $t$

$$
\begin{aligned}
q(u, 0, t)= & \left(c-c_{0}\right)+\left(c_{0}-c_{1}\right) \frac{s^{\prime}(t+W)}{s^{\prime}(t)}\left(\frac{\beta_{0}+u+2 s(t)}{\beta_{0}+u+s(t)+s(t+W)}\right)^{\alpha_{0}+1} \\
& +\mathbf{1}_{\left\{t<t_{1}(u+s(t))\right\}}\left\{\left(c-c_{0}\right)\left[1-\left(\frac{\beta_{0}+u+2 s(t)}{\beta_{0}+u+s(t)+s\left(t_{1}(u+s(t))\right)}\right)^{\alpha_{0}+1}\right]\right. \\
& +\left(c_{0}-c_{1}\right)\left(\beta_{0}+u+2 s(t)\right)^{\alpha_{0}+1}\left[\frac{1}{\left(\beta_{0}+u+s(t)+s(t+W)\right)^{\alpha_{0}+1}}\right. \\
& \left.\left.-\frac{1}{\left(\beta_{0}+u+s(t)+s\left(t_{1}(u+s(t))+W\right)\right)^{\alpha_{0}+1}}\right]\right\} .
\end{aligned}
$$




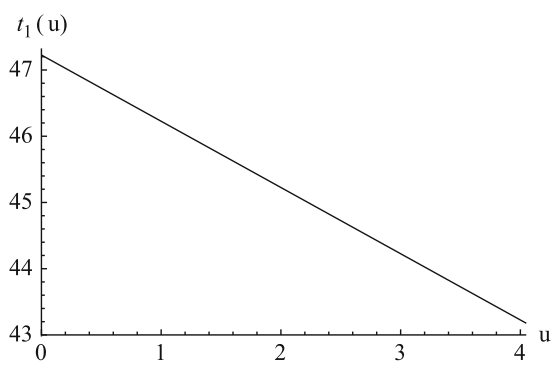

(a)

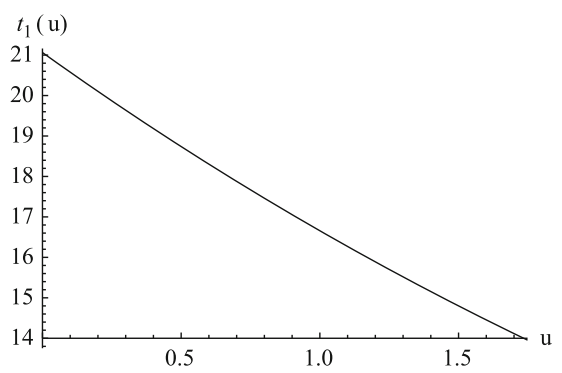

(c)

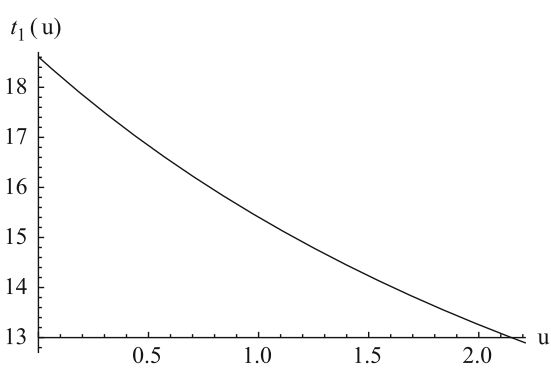

(b)

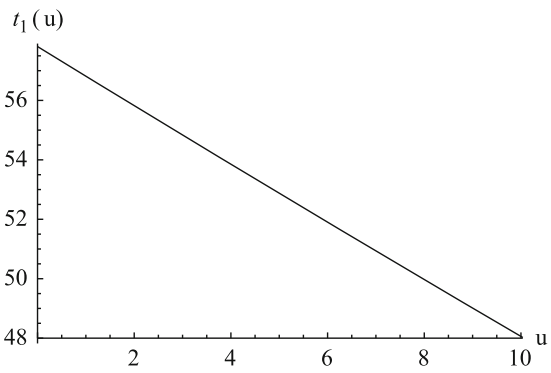

(d)

Fig. 1 In all above cases the parameters of the cost function and the a priori family are equal $c=$ 250, $c_{0}=260, c_{1}=-500, W=365.25, \alpha_{0}=3.4 \times 10^{-6}$ and $\beta_{0}=0.1$. a Exponential distribution, $s(t)=t, t_{0}=4.04944$. b (Shifted) Pareto distribution, $s(t)=\log (t+1), t_{0}=8.15055$. c Weibull distribution, $s(t)=\sqrt{t}, t_{0}=3.05799$. d Gompertz distribution, $s(t)=200\left(1-e^{-\frac{t}{200}}\right), t_{0}=10.299$

Let $t_{0}$ be the solution to the equation $q(0,0, t)=0$. Since $U_{t}=0$ when $H_{t}=0$, to describe the stopping region $\Gamma_{0}$, we need only $t_{0}$. Finally, the sets $\Gamma_{i}, i=0,1,2$, are of the following form

$$
\begin{aligned}
& \Gamma_{0}=\left\{(0,0, t): t \geq t_{0}\right\} \\
& \Gamma_{1}=\left\{(u, 1, t): 0 \leq u \leq s\left(t_{0}\right), t \geq t_{1}(u)\right\} \\
& \Gamma_{2}=\{(u, 2, t): u \geq 0, t \geq 0\} .
\end{aligned}
$$

Figure 1 shows the plots of the functions $t_{1}(u)$ for four different lifetime distributions. In the description of the plot, the value $t_{0}$, the numerical solution to the equation $q\left(0,0, t_{0}\right)=0$, is also given.

\section{Final remarks}

We have presented a generalization of the results obtained by Costantini and Spizzichino (1997). Our approach has allowed to solve the burn-in problem for a wider family of lifetime distributions. Considering the three-dimensional process in 
our model has led to more complicated value function. In the paper of Costantini and Spizzichino (1997) to obtain the solution, it was sufficient to derive the form of the value function of two arguments: $h \in\{0,1, \ldots, n\}$ and $y \in[0, \infty)$. In the general case considered, we had to derive the value function of three arguments: $h \in\{0,1, \ldots, n\}, u \in[0, \infty)$ and $t \in[0, \infty)$. Consequently, by the monotonicity property of the problem considered, in the paper of Costantini and Spizzichino (1997) the stopping region is represented by a sequence of $n$ numbers $y_{h}$ for each $h \in\{0,1, \ldots, n-1\}$. In our general case it is represented by a sequence of $n$ functions $t_{h}(u)$ for each $h \in\{0,1, \ldots, n-1\}$. Applying our approach to the exponential case leads to the solution given by $n$ linear functions instead of $n$ numbers. There is a direct relation between our solution and the solution presented in Costantini and Spizzichino (1997). It can be verified that in the case of $s(t)=t$, we get

$$
t_{h}(u)=\frac{y_{n-h}-u}{n-h} .
$$

From the above equation we can also see there is a slight difference in the interpretation of the results. Using the solution given in the paper of Costantini and Spizzichino (1997) we should stop the burn-in process if the total time on test $y=Y_{t}$ given by (5) exceeds the value $y_{h}$, where $h$ is the number of failures at time $t$. Taking advantage of our solution, we should stop the burn-in process if time $t$ exceeds $t_{h}(u)$, where $h$ is the number of failures at time $t$ and $u$ is the total time to failure $u=U_{t}$ given by (6), which is constant for a given $h$. Therefore, the description of the evolution of observation by the process $(U, H, t)$ leads to the solution of the problem considered which is simple to apply.

Acknowledgments The authors wish to thank the Referee whose constructive comments and valuable suggestions led to considerable improvements in the presentation.

Open Access This article is distributed under the terms of the Creative Commons Attribution License which permits any use, distribution, and reproduction in any medium, provided the original author(s) and the source are credited.

\section{References}

Arjas E (1989) Survival models and martingale dynamics. Scand J Stat 16:177-225

Aven T, Jensen U (1999) Stochastic models in reliability. Springer, New York

Barlow RE, Irony T (1993) The Bayesian approach to quality. In: Barlow RE, Clarotti CA, Spizzichino F (eds) Reliability and decision making, chap 13. Chapman \& Hall, London, pp 257-266

Barlow RE, Proschan F (1988) Handbook of statistics: quality control and reliability, vol 7, chap 9. Life distribution models and incomplete data. Elsevier Science Publishers B.V., Amsterdam, pp 225-249

Block HW, Savits TH (1997) Burn-in. Stat Sci 12(1):1-19

Bremaud P (1981) Point processes and queues, martingale dynamics. Springer, New York

Chang DS (2000) Optimal burn-in decision for products with an unimodal failure rate function. Eur J Oper Res 126(3):534-540

Clarotti CA, Spizzichino F (1990) Bayes burn-in procedures. Probab Eng Inf Sci 4:437-445

Costantini C, Spizzichino F (1997) Explicit solution of an optimal stopping problem: the burn-in of conditionally exponential components. J Appl Prob 34(1):267-282

de Finetti B (1937) La prévision: ses lois logiques, ses sources subjectives. Annales de l'institut Henri Poincaré 7:1-68 
Kececioglu D, Sun F (1997) Burn-in testing: its quantification and optimization. Prentice-Hall, Englewood Cliffs, NJ

Lai CD, Xie M (2006) Stochastic ageing and dependence for reliability, 1st edn. Springer Science + Business Media, New York

Leemis LM, Beneke M (1990) Burn-in models and methods: a review. IIE Trans Am Math Soc 22:172-180

Mi J (1995) Bathtub failure rate and upside-down bathtub mean residual life. IEEE Trans Reliab 44(3): 388-391

Peskir G, Shiryaev A (2006) Optimal stopping and free-boundary problems. Birkhauser, Basel

Runggaldier WJ (1993) On stochastic control concepts for sequential burn-in procedures. In: Barlow RE, Clarotti CA, Spizzichino F (eds) Reliability and decision making, chap 10. Chapman \& Hall, London, pp 211-232

Spizzichino F (1993) A unifying model for the optimal design of life-testing and burn-in. In: Barlow RE, Clarotti CA, Spizzichino F (eds) Reliability and decision making, chap 9. Chapman \& Hall, London, pp 189-210 